\title{
Resuscitative endovascular balloon occlusion of the aorta: current evidence
}

This article was published in the following Dove Medical Press journal: Open Access Emergency Medicine

\section{Lesley A Osborn' \\ Megan L Brenner ${ }^{2}$ \\ Samuel J Prater ${ }^{1}$ \\ Laura J Moore ${ }^{3}$}

'Department of Emergency Medicine, The University of Texas Health Science Center, McGovern Medical School, Houston, TX, USA; ${ }^{2}$ Department of Surgery, The University of California - Riverside, Moreno Valley, CA, USA; ${ }^{3}$ Department of Surgery, The University of Texas Health Science Center, McGovern Medical School, Houston, TX, USA
Correspondence: Lesley A Osborn Department of Emergency Medicine, The University of Texas Health Science Center - McGovern Medical School, 643I Fannin Street, JJL 45I, Houston, TX 77030, USA

Tel +I 7I35007878

Email Lesley.A.Osborn@uth.tmc.edu

\begin{abstract}
Resuscitative endovascular balloon occlusion of the aorta (REBOA) has recently gained popularity as a minimally invasive alternative to open aortic cross-clamping in the management of patients with non-compressible hemorrhage arising below the diaphragm. The purpose of this review is to provide a description of the technical aspects of REBOA use along with an overview of the current animal and clinical data regarding its use.
\end{abstract}

Keywords: REBOA, hemorrhagic shock, resuscitation

\section{Introduction}

Resuscitative endovascular balloon occlusion of the aorta (REBOA) has recently gained popularity as a minimally invasive alternative to open aortic cross-clamping in the management of patients with non-compressible hemorrhage arising below the diaphragm. The first clinical case series on the use of aortic balloon occlusion in the setting of abdominal trauma was written by Lieutenant Colonel Carl Hughes from the Korean War. ${ }^{1}$ After this initial case series, little was mentioned about the use of aortic occlusion balloons primarily due to limitations in the availability of intra-aortic balloon devices. For several decades, resuscitative thoracotomy (RT) with aortic occlusion via open cross-clamp was utilized for patients in extremis with non-compressible hemorrhage arising below the diaphragm. ${ }^{2}$ With the evolution of endovascular technology came the development of aortic occlusion balloons for use in the management of ruptured abdominal aortic aneurysms..$^{3-5}$ These first-generation aortic balloons were deployed via a 12 French sheath over a stiff guidewire under fluoroscopy and inflated above the rupture.

Ongoing experience from the military conflicts in Iraq and Afghanistan led to an increased interest in developing endovascular approaches to hemorrhage control, including the use of REBOA as an alternative to RT for the purpose of aortic occlusion. ${ }^{6}$ This led to military-civilian collaboration with the development of training courses tailored for trauma surgeons in the utilization of REBOA. ${ }^{7}$ Several early civilian adopters began to gain clinical experience with REBOA in the setting of both blunt and penetrating abdominal trauma. Early case series demonstrated that REBOA was effective at raising SBP in patients with hemorrhagic shock and was associated with a trend toward increased survival when compared with RT. ${ }^{89}$ With the renewed interest in the use of REBOA in the management of hemorrhage due to trauma has come further improvements in the catheters available, making its adoption and utilization even more widespread. The purpose of this review is to provide a description of the 
technical aspects of REBOA use along with an overview of the current animal and clinical data regarding its use.

\section{REBOA technique and indications}

REBOA requires an understanding of the anatomy of the aorta (Figure 1). Zone 1 of the aorta extends from the left subclavian artery to the celiac artery. Zone 2 continues from

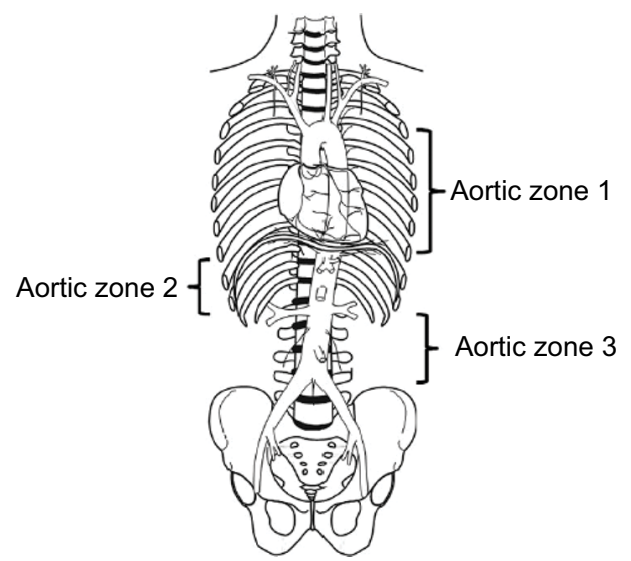

Figure I Aortic zones of occlusion from Stannard A, Eliason JL, Rasmussen TE. Resuscitative endovascular balloon occlusion of the aorta (REBOA) as an adjunct for hemorrhagic shock. J Trauma, vol 7I, issue 6, pages 1869-1872." the celiac artery to the renal artery. Zone 3 extends from the origin of the lowest renal artery to the aortic bifurcation (infrarenal aorta). Zone 1 occlusion is utilized in patients in cardiac arrest or those in hemorrhagic shock with evidence of non-compressible hemorrhage arising below the diaphragm. Zone 2 is considered to be a no-occlusion zone. Zone 3 occlusion is reserved for patients without evidence of intra-abdominal hemorrhage but with evidence of a pelvic fracture.

A clinical algorithm outlining the clinical decisionmaking for REBOA utilization is presented in Figure 2. ${ }^{10}$ Prior to making the decision to utilize REBOA, it is imperative that a chest X-ray (CXR) be obtained to rule out a major thoracic vascular injury or other intra-thoracic pathology as the source of the patient's hemodynamic compromise (tension pneumo- or hemothorax). If CXR is suspicious for aortic injury consistent with mechanism (widened mediastinum, apical cap, and depressed left mainstem bronchus) then REBOA is contraindicated. Patients in arrest are unlikely to undergo CXR but tube or finger thoracostomy to rule out tension pneumothorax and massive hemothorax should be performed. The focused assessment with sonography for

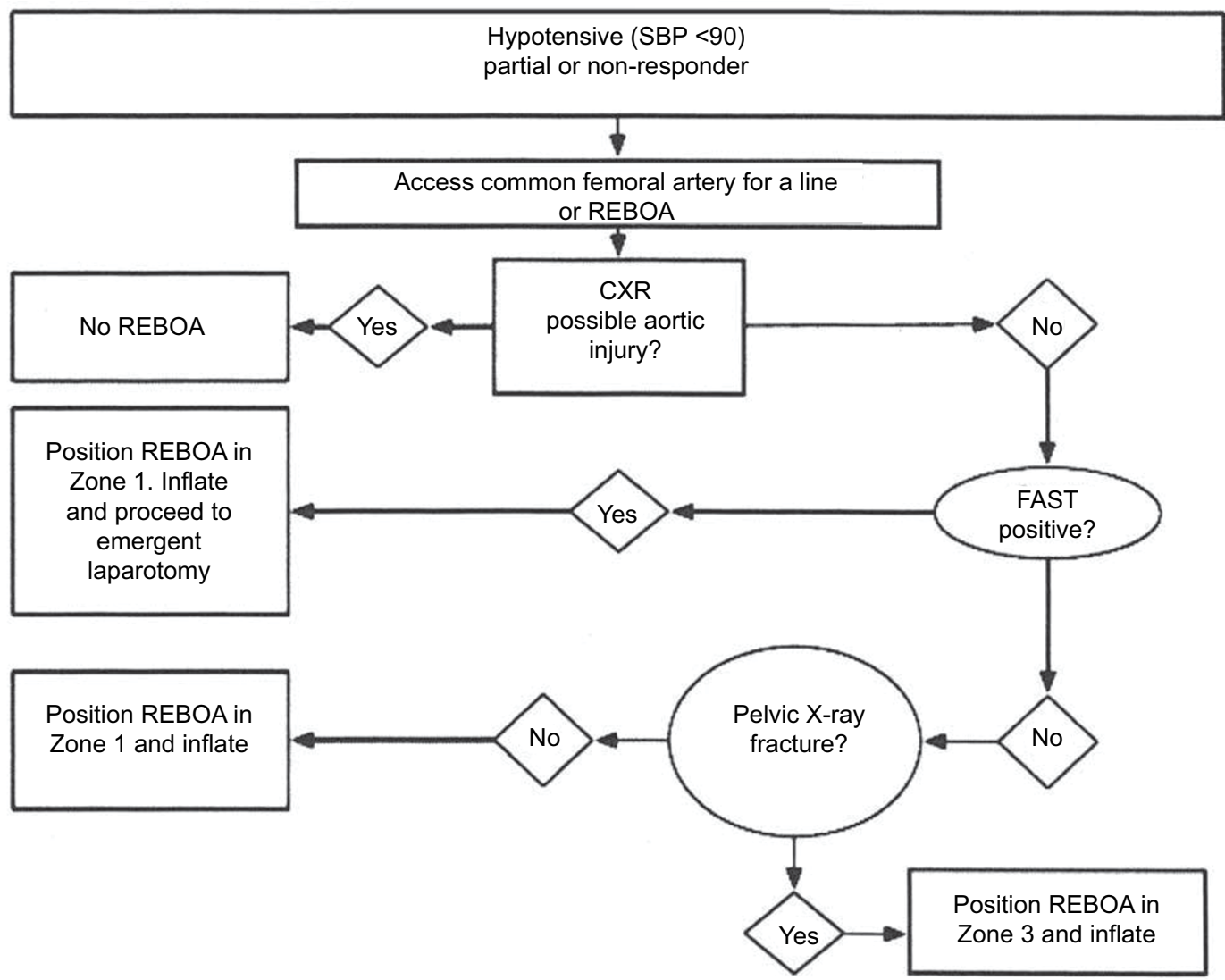

Figure 2 REBOA algorithm from Brenner M, Hoehn M, Pasley J, Dubose J, Stein D, Scalea T. Basic endovascular skills for trauma course: bridging the gap between endovascular techniques and the acute care surgeon. L Trauma Acute Care Surg, vol 77, issue 2, pages 286-29I.10

Abbreviations: CXR, chest X-ray; FAST, focused assessment with sonography for trauma; REBOA, resuscitative endovascular balloon occlusion of the aorta. 
trauma (FAST) exam, which both emergency physicians and trauma surgeons are facile with, is also utilized to assist with locating the source of hemorrhage within the abdomen, along with ruling out pericardial tamponade. Patients with hypotension (defined as SBP $<90 \mathrm{mmHg}$ ) not responsive to initial blood product resuscitation and with a positive abdominal FAST exam would be candidates for Zone 1 occlusion. Patients who are hypotensive with a negative FAST exam and a pelvic fracture would be candidates for Zone 3 occlusion.

A comprehensive description of REBOA technique is beyond the scope of this review. Additional information about the technical aspects of REBOA has been published by Stannard et al. ${ }^{11}$ Clinicians interested in implementing REBOA into their clinical practice should seek formal training via one of the available REBOA courses as described ahead. REBOA can be divided into the following steps:

1. Arterial access with insertion of arterial sheath (12 vs 7 French depending on the catheter used)

2. Insertion of REBOA catheter with balloon positioning at appropriate aortic zone

3. Balloon inflation

4. Balloon deflation

5. REBOA catheter removal

6. Arterial sheath removal

Arterial access has been shown to be the critical, often rate limiting factor in the utilization of REBOA. The common femoral artery (CFA) should be accessed under ultrasound guidance if available. External anatomical landmarks can assist in accessing the CFA when ultrasound is not available, though this is less ideal. Arterial cut-down also allows for direct visualization of the CFA and may be the more reliable method of access in patients already in arrest. ${ }^{12}$ However, a trauma or vascular surgeon is more likely to be familiar with this technique. Also, it may increase the time to aortic occlusion when performed by those unfamiliar with this procedure. Inadvertent cannulation of the superficial femoral artery has resulted in significant vascular injury and should be avoided. ${ }^{13}$ REBOA has been shown to be a faster method to aortic occlusion than RT after arterial access is obtained, further highlighting the importance of rapid, successful CFA access. ${ }^{12}$ However, for patients already in cardiac arrest, it is significantly more difficult to obtain reliable percutaneous arterial access. Rapid conversion to open arterial cut-down or consideration for performing RT is recommended for patients in cardiac arrest.

After the appropriately sized sheath has been placed, the aortic zone of occlusion (Zone 1 or 3 ) must be decided based on the clinical algorithm discussed previously. External landmarks are used to estimate appropriate catheter insertion depth. Confirmation of appropriate balloon location via digital X-ray should be performed prior to balloon inflation. At our center, this is done by performing a CXR or pelvic X-ray in the emergency department (ED). The catheter should be seen on the patient's left side adjacent to the spine. Zone 1 is confirmed by visualizing the balloon above the diaphragm (Figure 3). Confirmation of the device in Zone 3 is seen on pelvis X-ray as being in the vertical position at approximately the L2 or L3 vertebrae (Figure 4).

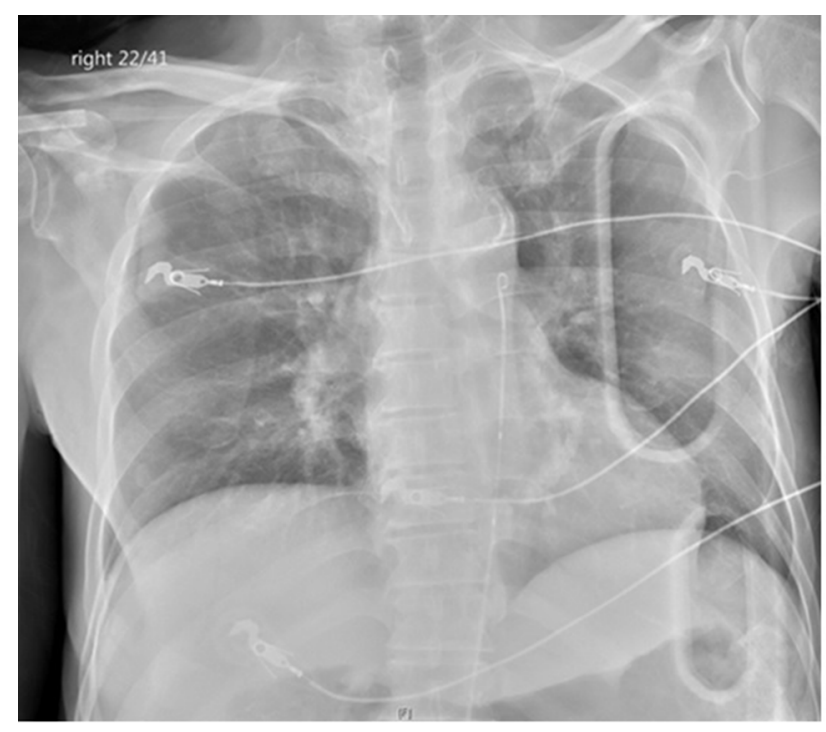

Figure 3 X-ray imaging of Zone I REBOA.

Abbreviation: REBOA, resuscitative endovascular balloon occlusion of the avorta

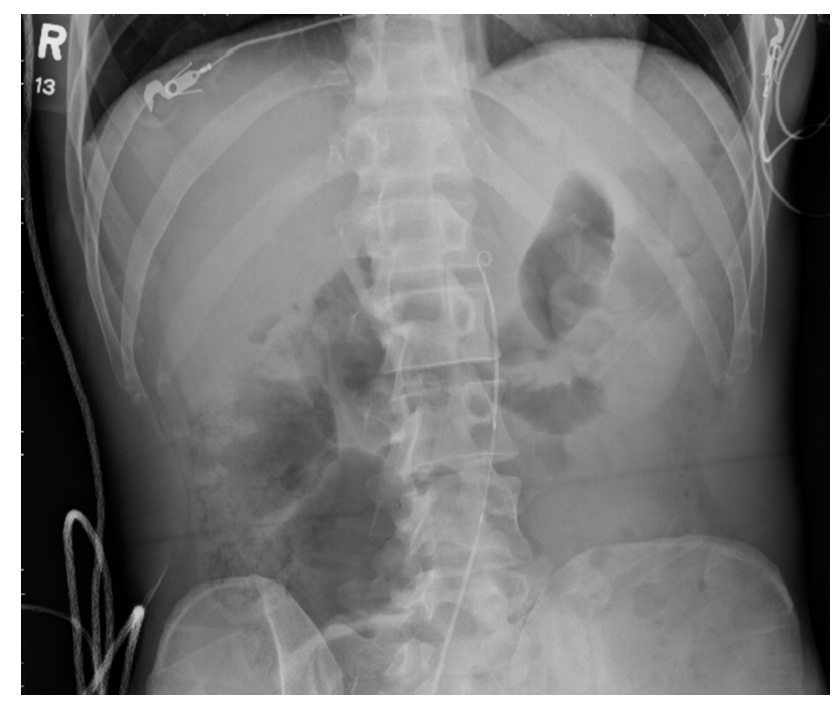

Figure 4 X-ray imaging of Zone 3 REBOA.

Abbreviation: REBOA, resuscitative endovascular balloon occlusion of the aorta. 
The volume of inflation varies among devices and is dependent on zone of occlusion, with Zone 1 inflation volume typically being larger than those required in Zone 3 due to differences in aortic diameter. Transduction of arterial pressure from the REBOA catheter during balloon inflation is helpful in determining optimal balloon inflation volume. In addition, monitoring for loss of the contralateral femoral pulse by palpation or Doppler indicates sufficient balloon inflation has occurred. Once the device is deployed in the desired zone, securing the device is critical to prevent potential complications from balloon migration. Finally, the time of occlusion should be documented. Following balloon inflation, the patient should proceed emergently to either the operating room for exploratory laparotomy or the interventional/hybrid suite for angioembolization. It is important to remember that REBOA is only a temporizing measure meant to sustain perfusion to the heart and brain en route to rapid, definitive hemorrhage control. It is imperative that clinicians utilizing REBOA be aware of the potential complications related to the level and duration of balloon inflation. Over inflation of the balloon can result in either balloon or blood vessel rupture. In a recent systematic review, Morrison et al identified three reported deaths that were directly attributed to balloon over-inflation with resultant aortic rupture. ${ }^{14}$

Once definitive hemorrhage control has been obtained, balloon deflation can occur. This requires coordination with the anesthesiologist in the operating room or hybrid suite to optimize volume status. In our experience, incremental balloon deflation is typically required in order to avoid cardiovascular collapse. Once the balloon has been deflated, the REBOA catheter can be removed from the sheath. After the catheter has been removed, it is critical to assess distal perfusion in bilateral lower extremities. Diminished or absent distal pulses in either lower extremity following REBOA warrant immediate investigation with angiography to identify any potential vascular complications arising from either the sheath or the catheter.

The technique for sheath removal is dependent on the size of sheath inserted. The 12 French sheaths must be removed via open cut-down with repair of the arteriotomy site and should be removed at the index operation. The 7 French sheaths do not require cut-down with arterial repair unless vascular compromise has been identified. At our institution, 7 French sheaths are pulled in the intensive care unit after we have verified correction of coagulopathy. Pressure is then held for 30 minutes at the puncture site followed by 6 hours of bed rest. Neurovascular checks of the lower extremity are also performed every hour for the first 24 hours after sheath removal. An ultrasound of the arterial access site is performed 48 hours after sheath removal to assess for pseudoaneurysm formation.

\section{Current REBOA data}

\section{Animal studies}

Multiple animal studies evaluating REBOA utilization have been performed. These studies are all short term, lethal hemorrhagic shock models focusing primarily on balloon inflation times and the associated physiological consequences. Markov et al studied the effect of 30 vs 90 minutes of occlusion time in a class IV hemorrhagic shock model in swine. They found that Zone 1 REBOA inflation improved mean central aortic pressure but resulted in significant elevation in serum lactate levels compared with swine in hemorrhagic shock without REBOA. ${ }^{15}$ Morrison et al demonstrated a clear survival advantage with both continuous and intermittent REBOA in their study using an abdominal hemorrhage model in swine (25\% vs $37.5 \%$ vs $100 \%$ mortality). ${ }^{16}$ White et al compared RT with open aortic cross-clamping to Zone 1 REBOA in a class IV hemorrhagic shock model and found that REBOA increased central perfusion pressures with less physiologic disturbance than RT with open aortic clamping. ${ }^{17}$ Kuckelman et al evaluated the potential benefits of intermittent vs full occlusion at Zone 1 in another class IV hemorrhagic shock model in swine. The authors concluded that intermittent occlusion provided similar hemorrhage control with fewer physiologic consequences as demonstrated by decreased acidosis and lower resuscitation requirements in the intermittent occlusion group. ${ }^{18}$ In another swine model, Tibbits et al evaluated the hemodynamic consequences associated with Zone 1 vs 3 occlusion. They found that Zone 3 REBOA provided minimal hemodynamic support compared with Zone 1 REBOA. They also noted less ischemic burden and instability upon reperfusion with Zone 3 vs 1 inflation. These findings suggest that, in cases of impending hemodynamic collapse, Zone 1 REBOA placement may be more efficacious than Zone 3 regardless of injury pattern. ${ }^{19}$

\section{Human use data}

The current literature regarding REBOA utilization is increasing but has been limited to large case series. Brenner et al published the first modern day case series in the USA reporting on use of the 12 French system on six patients from two level 1 trauma centers. ${ }^{8}$ REBOA placement resulted in a mean increase in the BP of $55 \mathrm{mmHg}$ and no hemorrhage-related mortality. Moore et al published the first comparison of 
REBOA as an alternative to RT for non-compressible truncal hemorrhage arising below the diaphragm and demonstrated an improved overall survival and fewer early deaths due to hemorrhage in patients undergoing REBOA. ${ }^{9}$ The American Association for the Surgery of Trauma Aortic Occlusion for Resuscitation in Trauma and Acute Care Surgery registry has published similar findings, with a trend toward improved survival in patients undergoing REBOA compared with RT. The survival benefit is most pronounced in patients not undergoing cardiopulmonary resuscitation at the time of aortic occlusion. Additionally, REBOA was found to result in a more consistent improvement in hemodynamic stability compared with RT.

The duration of occlusion remains an important factor when considering deployment of a REBOA device. Animal data have suggested that inflation time of 30 minutes at Zone 1 are well tolerated but 60 minutes of Zone 1 occlusion results in significant physiologic derangements that preclude any potential benefit. ${ }^{20}$ With a goal of $<60$ minutes of occlusion in Zone 1 and $\sim 90$ minutes in Zone 3, rapid access to definitive trauma/vascular surgical management is critical. The goal of REBOA is to improve survival, not prolong time to death. There exists the potential to expand this window of time with the use of partial REBOA, though further studies are necessary to define this technique and its exact parameters. Partial occlusion of the aorta can be achieved by not inflating the balloon to the point of aortic occlusion..$^{21,22}$

Outside of the USA, REBOA has also become a favorable option in the Japanese EDs, including implementing training on REBOA for emergency medicine (EM) residents as part of their education requirements prior to graduation. ${ }^{23}$ The Japanese REBOA experience differs somewhat from the REBOA experience in the USA. While surgeons are involved in REBOA deployment, many of the REBOA deployments in Japan are performed by EM physicians having additional training in interventional radiology. Another significant difference from the US practice is that the Japanese uniformly place REBOA in Zone 1 regardless of the pattern of injury. Sato et al published a case series of 24 patients in which REBOA was placed in four Japanese EDs that did not have immediate access to a trauma surgeon. In this series, the median balloon inflation time was 65 minutes at Zone 1 with a $50 \%$ mortality rate at 24 hours. Five of the 12 survivors at 24 hours experienced complications, including one case of mesenteric ischemia, one case of lower extremity ischemia, and three cases of REBOA inflation in patients with thoracic aortic injuries. ${ }^{24}$ Kunitatsu et al performed a retrospective review of 44 hemorrhagic shock patients who underwent emergency laparotomy for intra-abdominal hemorrhage over a 6-year period at a single center. Of these patients, 19 underwent REBOA during initial resuscitation to control massive intra-abdominal hemorrhage. Balloon inflation resulted in a significant improvement in SBP, with mean increase from $75 \pm 10$ $\mathrm{mmHg}$ to $124 \pm 22 \mathrm{mmHg}$ and with an overall survival rate of $77.3 \%$ and no complications associated with REBOA. ${ }^{25}$ In another large case series from Japan, Matsumura et al reported on 109 REBOA cases from 23 centers. They noted that survivors had a higher pre-occlusion SBP, earlier initial arterial access, and shorter balloon inflation times. ${ }^{26}$ The median time of arrival to arterial access was 25 minutes in survivors vs 47 minutes in non-survivors. These findings further emphasize the importance of rapid, accurate central arterial access for patients undergoing REBOA, as discussed previously. Achieving early arterial access with an 18-gauge arterial line placed in the CFA allows for rapid upsizing to a 7 or 12 French sheath for REBOA deployment. The difficulty in obtaining CFA access increases exponentially as a patient's hemodynamic status deteriorates.

With the advent of smaller, wire-free devices, the utilization of REBOA in the combat casualty setting has increased. Manley et al presented a case series of four wartime injured patients with torso gunshot or fragmentation wounds, hemoperitoneum, and class IV shock that were cared for by a US Air Force Special Operations Surgical Team in an austere location. ${ }^{27}$ Three patients underwent Zone 1 deployment and one patient underwent Zone 3 deployment. All patients experienced an improvement in their SBP with balloon inflation and there were no access or REBOA-related complications. All patients survived to be transported to the next echelon of care in stable condition. This was followed by a larger military case series by Northern et al describing 20 military combat casualties with injuries from explosions and gunshot wounds. ${ }^{28}$ All patients were in hemorrhagic shock with mean initial heart rate of $129 \mathrm{bpm}$ and mean initial SBP of $71 \mathrm{mmHg}$. Aortic occlusion was achieved with REBOA catheter placement in Zone $1(n=17)$ and Zone $3(n=2)$. SBP increased to an average of $56 \mathrm{mmHg}$ with aortic occlusion. There were no access-related site complications and all 20 patients survived to achieve transport to the next level of care.

Pre-hospital use of REBOA has also recently been described in the civilian trauma setting. The London Air Ambulance Physician Paramedic Team described the first civilian pre-hospital REBOA in a 32-year-old male that had fallen 15 feet with an obvious pelvic fracture. ${ }^{29}$ Zone 
3 deployment resulted in improved hemodynamics and the patient was transported to a trauma center where he underwent angioembolization of the pelvis. He was subsequently discharged on hospital day 52. Recently, Lamhaut et al described the first civilian pre-hospital Zone 1 REBOA deployment by the Service d'Aide Medicale Urgente in Paris, France. This is a physician delivered pre-hospital service that has experience performing extracorporeal life support (extracorporeal cardiopulmonary resuscitation) in the field. ${ }^{30}$ They placed a Zone 1 REBOA in a female patient undergoing CPR with presumed abdominal hemorrhage. The balloon was inflated within 17 minutes of physician arrival on the scene and the patient was in the operating theater within 36 minutes of balloon inflation. The patient survived her initial resuscitation but was found to have metastatic cancer and transitioned to palliative care. The authors noted that it is "imperative that REBOA is developed within a system that can rapidly transport to definitive repair". ${ }^{30}$

One of the challenges of REBOA placement in the prehospital setting is the lack of imaging capability to confirm REBOA position prior to balloon inflation. Wessels et al recently described the experimental use of radiofrequency identification to determine REBOA placement in a perfused cadaver model. ${ }^{31}$ Ogura et al described their protocol for using ultrasound to determine REBOA catheter placement using a wire-based system in conjunction with radial arterial line monitoring. ${ }^{32}$ The authors have limited anecdotal experience with using ultrasound to visualize the balloon inflated in Zone 3 but ultrasound visualization of Zone 1 placement is technically challenging.

\section{REBOA utilization in non-traumatic hemorrhage}

The non-traumatic hemorrhagic shock patient is another potential arena that has yet to be further explored with regard to endovascular aortic occlusion. Recent promising data out of a single center case series of eleven patients in hemorrhagic shock at a large tertiary care center in the USA describes success in placing this device in patients with non-trauma-related intra-abdominal hemorrhage. ${ }^{33}$ Of the eleven patients, $64 \%$ were in arrest due to hemorrhage prior to REBOA placement. The primary causes of intra-abdominal hemorrhage were reported to be massive upper gastrointestinal tract bleeding and ruptured visceral aneurysms. Despite the high proportion of patients in arrest, the in-hospital survival rate was $64 \%$ and there were no REBOA-related complications reported. Shigesato et al reported successful use of REBOA in resuscitating a patient in cardiac arrest from hemorrhagic shock due to a duodenal ulcer bleed. ${ }^{34}$ Despite receiving 20 minutes of CPR prior to return of spontaneous circulation, this patient survived and was discharged without neurological deficit on hospital day 31. These examples open the door for potential expansion of indications for REBOA in other patient populations.

A recent article by Dogan et al in Sweden demonstrates the potential for REBOA in non-traumatic cardiac arrest patients. ${ }^{35}$ Though this was a small animal model study, it raises the hypothesis of the utility of Zone 1 occlusion in augmenting arterial pressure, similar to an intra-aortic balloon pump, and improving cardiac and cerebral perfusion pressures during cardiac arrest resuscitation. Identifying the appropriate patient population (minimal downtime, arrest due to ventricular fibrillation, age qualifications, etc) will be critical to implementing this in the non-traumatic cardiac arrest patient. This would require the support of a tertiary center and resuscitation specialists with the capability of performing percutaneous coronary intervention during arrest and/or initiating extracorporeal membrane oxygenation. ${ }^{35,36}$

The utilization of REBOA in the management of peri- and post-partum hemorrhage is being reported with increased frequency. With peri- and post-partum hemorrhage remaining a major cause of maternal and fetal death worldwide, utilizing a device that limits hemorrhage prophylactically, intraoperatively or post-partum, could be life-saving and reduce the large volume blood transfusions currently necessary in certain high-risk deliveries. These scenarios include placenta percreta, placenta accreta, and placenta previa. Prophylactic insertion of iliac artery occlusion balloons has been described in the setting of elective cesarean section for woman with malignant placental implantation. However, occlusion at the level of the common iliac artery does not occlude the ovarian arteries, which arise directly off of the infra-renal abdominal aorta. Studies by Ordonez et al and Sun et al demonstrate the feasibility of using REBOA prophylactically in cesarean section patients with adherent placentas and the potential to decrease blood loss during these high-risk procedures. ${ }^{37,38}$ Currently, a much-needed prospective randomized trial is in the planning stages to examine prophylactic abdominal aortic occlusion in patients with placenta accrete. ${ }^{39}$

Another element that has been mentioned in the obstetrics literature and might be applicable to other medical and trauma scenarios, is the use of aortic occlusion in patients who opt out of blood transfusions, like seen with many Jehovah's Witnesses. ${ }^{40}$ Expansion of REBOA application into areas with limited availability of blood products could be potentially life-saving. However, austere environments and less 
developed health care systems have their own challenges, such as lack of access to surgeons with vascular expertise needed to address potential complications. Therefore, available resources in addition to cost must be considered when implementing a novel device like REBOA. Though further data are necessary to define the proper indications for implementation of REBOA in the non-trauma patient, the aforementioned literature shines light on a promising future.

\section{Complications and pitfalls of REBOA}

A variety of complications related to REBOA utilization have been reported in the literature. Ischemia secondary to prolonged balloon inflation, aortic or iliac artery rupture or dissection, arterial vascular thrombosis, pseudoaneurysm formation, and limb amputation have all been reported. As expected, more vascular complications have been observed with the larger 14 or 12 French systems but complications have occurred with the 7 French systems as well. Taylor et al described their experience with 48 patients who underwent REBOA (38 with a 14 French system and 10 with a 7 French System). ${ }^{13}$ Of the 24 patients who survived until removal of the 14 French sheath, 19 had primary repair of the arteriotomy without vascular complication. The other five patients required additional vascular procedures to repair the arteriotomy and restore distal flow. Among these five patients, two had inadvertent but incorrect insertion of the 14 French sheath into the superficial femoral artery. There were no vascular complications of sheath removal with the 7 French system. No lower-extremity amputations were attributed to REBOA in this series.

In a recent review by Ribeiro et al, REBOA-related complications are described. Major complications of REBOA catheter insertion are related to vessel injuries, including aortic dissection, rupture, perforation, embolization, air emboli, and peripheral ischemia. ${ }^{40}$ Ordonez et al reported their case series of 27 patients undergoing REBOA via a 10 French system. They reported an $18 \%$ incidence of REBOArelated complications with three patients having arterial access-related issues requiring open operative repair, and two patients with visceral ischemia (gastric and right colon). ${ }^{41}$ Saito et al reported on their experience with 24 REBOA cases using a 10 French system from Japan. In this series, a $12.5 \%$ complication rate was reported with three patients requiring lower extremity amputations due to vascular accesssite complications. ${ }^{42}$ In another Japanese series, Matsumura reported on 142 patients that underwent REBOA with either a larger sheath (12-14 French) or smaller sheath (7-8 French). Two patients in the small sheath group developed complications (one arterial dissection, one massive hematoma). Three patients in the large sheath group developed complications with one patient developing arterial thrombus and two patients developing distal ischemia requiring limb amputation. As anticipated, fewer arterial complications are being reported with the use of smaller, 7 French sheaths. A Japanese case series by Teeter et al reported no access-related complications in 33 patients undergoing Zone 1 REBOA via a 7 French system. ${ }^{43}$

Because of the potential for major vascular complications to occur, REBOA should be performed by a clinician trained in REBOA with knowledge of the potential complications in conjunction with an acute care surgeon or an interventionalist (vascular surgeon or interventional radiologist) trained in REBOA. Additionally, in order to resolve possible vascular complications, a vascular surgeon must be available. Identifying these individuals is a critical component of implementing a successful REBOA practice at any institution.

\section{Skills and training for REBOA}

Surgical exposure of the CFA is a skill needed for successful use of REBOA should percutaneous access not be possible. Early clinical experience demonstrates that almost half of patients required surgical cut-down for access, although subsequent series and increasing experience with percutaneous access is reducing this need. ${ }^{44,45}$ EM physicians are quite facile at percutaneous ultrasound-guided CFA cannulation, which could negate the need for surgical cut-down. The largest limitation for use of REBOA in the ED is lack of definitive hemorrhage control. Due to the intolerance of aortic occlusion for lengthy periods of time (particularly at Zone 1), REBOA is not advocated unless surgical and/ or interventional resources are immediately available. ${ }^{46}$ Another relative limitation for use of REBOA in the ED is the inability to repair complications, particularly those that require immediate attention, if resources are not available to provide the necessary assistance. It is more the location where REBOA occurs, rather than the title or training of the proceduralist, that is critical in assuring good outcomes for these patients. The military experience, as well as some EM physicians working with trauma teams in civilian trauma centers, demonstrate that EM physicians can perform the procedure with good outcomes as part of a surgical team when hemorrhage control is immediately available.

Training courses are available for REBOA education across the USA and abroad. As with any skills simulation training, it is critical to learn from those with significant clinical experience, particularly when endovascular and open 
surgical skills are not part of a physician's routine practice. Participation at any REBOA course does not confer institutional credentialing to perform the procedure. In some highvolume centers, completion of an accredited course is required for acute care surgeons to apply for privileges, ${ }^{10}$ which has shown to result in safe performance of the procedure and continued use of REBOA in clinical practice. ${ }^{47}$ EM physicians who have taken the BEST course have also demonstrated proficiency with REBOA in an advanced military system with surgical support. ${ }^{28}$ Conversely, some institutions require no formal training and have demonstrated safe use of REBOA by acute care surgeons in environments with experienced surgical leadership. ${ }^{48}$ Each institution is responsible for determining requirements for clinical REBOA implementation.

\section{Recommendations for "best case scenario" use in EM}

Optimal conditions for deployment of REBOA are a coordinated team approach in trauma centers with readily available access to trauma and vascular surgery. EM physicians are well-suited partners in the management of hemorrhagic shock given their expertise in ultrasound-guided vascular access, emergent procedures, and advanced airway management. Patients in hemorrhagic shock present a unique challenge in obtaining adequate venous and arterial access; ultrasound guidance is often a critical skill and early arterial access of the CFA with an 18-gauge catheter in these patients can prove to be a shrewd maneuver. The 18-guage catheter can easily be upsized to a 7 or 12 French sheath. EM physicians are adept with ultrasound-guided access making them ideal partners in obtaining critical arterial access and placement of a REBOA catheter in conjunction with the trauma surgeon or even before the trauma surgeon arrives. This is especially important in higher volume trauma centers where the trauma surgeon may be temporarily encumbered. Additionally, mass casualty situations would lend themselves to partnering with EM physicians for initiation of a REBOA catheter for temporary hemorrhage control while awaiting definitive control. Patients in cardiac arrest are less ideal candidates given limited experience by EM physicians with arterial cutdown procedures when compared with trauma and vascular surgeons. Patients requiring transfer out to another facility for definitive hemorrhage control are not ideal candidates due to the need for prolonged occlusion, especially in Zone 1. Additionally, lack of familiarity with REBOA of EMS personnel or even receiving center personnel would also prove to be prohibitive to placement of a REBOA catheter prior to transfer. Doing so would require short transport times and a comprehensive team of physicians competent in REBOA catheter placement and management to be present during all phases of the transfer process. A report from a mature, coordinated system has described the implementation of a REBOA program at outlying facilities where two patients were transferred after REBOA; in one case, the physician accompanied the patient to the tertiary care center in order to manage the catheter and continue resuscitation. ${ }^{49}$ This could likely only be accomplished in a very small number of comprehensive trauma systems.

\section{Conclusion}

REBOA is a useful adjunct in the emergent management of non-compressible truncal hemorrhage arising below the diaphragm. The use of REBOA provides for temporary hemorrhage control and improved hemodynamics until definitive surgical or endovascular hemorrhage control can be achieved.

\section{Disclosure}

Megan L Brenner is a Clinical Advisory Board Member of Prytime Medical Inc. The authors report no other conflicts of interest in this work.

\section{References}

1. Hughes CW. Use of an intra-aortic balloon catheter tamponade for controlling intra-abdominal hemorrhage in man. Surgery. 1954;36(1):65-68.

2. Ledgerwood AM, Kazmers M, Lucas CE. The role of thoracic aortic occlusion for massive hemoperitoneum. J Trauma. 1976;16(08):610-615.

3. Malina M, Holst J. Balloon control for ruptured AAAs: when and when not to use? J Cardiovasc Surg (Torino). 2014;55:161-167.

4. Philipsen TE, Hendriks JM, Lauwers P, et al. The use of rapid endovascular balloon occlusion in unstable patients with ruptured abdominal aortic aneurysm. Innovations (Phila). 2009;4(2):74-79.

5. Malina M, Veith F, Ivancev K, Sonesson B. Balloon occlusion of the aorta during endovascular repair of ruptured abdominal aortic aneurysm. J Endovasc Ther. 2005;12(5):556-559.

6. Rasmussen TE, Dubose JJ, Asensio JA, et al. Tourniquets, vascular shunts, and endovascular technologies: esoteric or essential? A report from the 2011 AAST Military Liaison Panel. JTrauma Acute Care Surg. 2012;73(1):282-285.

7. Villamaria CY, Eliason JL, Napolitano LM, Stansfield RB, Spencer JR, Rasmussen TE. Endovascular Skills for Trauma and Resuscitative Surgery (ESTARS) course: curriculum development, content validation, and program assessment. J Trauma Acute Care Surg. 2014;76(4):929-935.

8. Brenner ML, Moore LJ, Dubose JJ, et al. A clinical series of resuscitative endovascular balloon occlusion of the aorta for hemorrhage control and resuscitation. J Trauma Acute Care Surg. 2013;75(3):506-511.

9. Moore LJ, Brenner M, Kozar RA, et al. Implementation of resuscitative endovascular balloon occlusion of the aorta as an alternative to resuscitative thoracotomy for noncompressible truncal hemorrhage. $J$ Trauma Acute Care Surg. 2015;79(4):523-532.

10. Brenner M, Hoehn M, Pasley J, Dubose J, Stein D, Scalea T. Basic endovascular skills for trauma course: bridging the gap between endovascular techniques and the acute care surgeon. J Trauma Acute Care Surg. 2014;77(2):286-291. 
11. Stannard A, Eliason JL, Rasmussen TE. Resuscitative endovascular balloon occlusion of the aorta (REBOA) as an adjunct for hemorrhagic shock. J Trauma. 2011;71(6):1869-1872.

12. Romagnoli A, Teeter W, Pasley J, et al. Time to aortic occlusion: it's all about access. J Trauma Acute Care Surg. 2017;83(6):1161-1164.

13. Taylor JR, Harvin JA, Martin C, Holcomb JB, Moore LJ. Vascular complications from resuscitative endovascular balloon occlusion of the aorta: life over limb?. J Trauma Acute Care Surg. 2017;83(1 Suppl 1):S120-S123.

14. Morrison JJ, Galgon RE, Jansen JO, Cannon JW, Rasmussen TE, Eliason JL. A systematic review of the use of resuscitative endovascular balloon occlusion of the aorta in the management of hemorrhagic shock. J Trauma Acute Care Surg. 2016;80(2):324-334.

15. Markov NP, Percival TJ, Morrison JJ, et al. Physiologic tolerance of descending thoracic aortic balloon occlusion in a swine model of hemorrhagic shock. Surgery. 2013;153(6):848-856.

16. Morrison JJ, Ross JD, Houston R, Watson JD, Sokol KK, Rasmussen TE. Use of resuscitative endovascular balloon occlusion of the aorta in a highly lethal model of noncompressible torso hemorrhage. Shock 2014;41(2):130-137.

17. White JM, Cannon JW, Stannard A, Markov NP, Spencer JR, Rasmussen TE. Endovascular balloon occlusion of the aorta is superior to resuscitative thoracotomy with aortic clamping in a porcine model of hemorrhagic shock. Surgery. 2011;150(3):400-409.

18. Kuckelman JP, Barron M, Moe D, et al. Extending the golden hour for zone 1 resuscitative endovascular balloon occlusion of the aorta: improved survival and reperfusion injury with intermittent versus continuous resuscitative endovascular balloon occlusion of the aorta of the aorta in a porcine severe truncal hemorrhage model. J Trauma Acute Care Surg. 2018;85(2):318-326.

19. Tibbits EM, Hoareau GL, Simon MA, et al. Location is everything: the hemodynamic effects of REBOA in zone 1 versus zone 3 of the aorta. J Trauma Acute Care Surg. 2018;85(1):101-107.

20. Reva VA, Matsumura Y, Hörer T, et al. Resuscitative endovascular balloon occlusion of the aorta: what is the optimum occlusion time in an ovine model of hemorrhagic shock? Eur J Trauma Emerg Surg. 2018;44(4):511-518.

21. Davidson AJ, Russo RM, Ferencz SE, et al. Incremental balloon deflation following complete resuscitative endovascular balloon occlusion of the aorta results in steep inflection of flow and rapid reperfusion in a large animal model of hemorrhagic shock. J Trauma Acute Care Surg. 2017;83(1):139-143.

22. Johnson MA, Williams TK, Ferencz SE, et al. The effect of resuscitative endovascular balloon occlusion of the aorta, partial aortic occlusion and aggressive blood transfusion on traumatic brain injury in a swine multiple injuries model. J Trauma Acute Care Surg. 2017;83(1):61-70.

23. Gamberini E, Coccolini F, Tamagnini B, et al. Resuscitative endovascular balloon occlusion of the aorta in trauma: a systematic review of the literature. World J Emerg Surg. 2017;12:42.

24. Sato R, Kuriyama A, Takaesu R, et al. Resuscitative endovascular balloon occlusion of the aorta performed by emergency physicians for traumatic hemorrhagic shock: a case series from Japanese emergency rooms. Crit Care. 2018;22(1):103.

25. Kunitatsu K, Ueda K, Iwasaki Y, et al. Outcomes of abdominal trauma patients with hemorrhagic shock requiring emergency laparotomy: efficacy of intra-aortic balloon occlusion. Acute Med Surg. 2016;3(4):345-350.

26. Matsumura Y, Matsumoto J, Kondo H, et al; DIRECT-IABO Investigator. Early arterial access for resuscitative endovascular balloon occlusion of the aorta is related to survival outcome in trauma. J Trauma Acute Care Surg. 2018;85(3):507-511.

27. Manley JD, Mitchell BJ, DuBose JJ, Rasmussen TE. A modern case series of resuscitative endovascular balloon occlusion of the aorta (REBOA) in an out-of-hospital, combat casualty care setting. $J$ Spec Oper Med. 2017;17(1):1-8.
28. Northern DM, Manley JD, Lyon R, et al. Recent advances in austere combat surgery: use of aortic balloon occlusion as well as blood challenges by special operations medical forces in recent combat operations. J Trauma Acute Care Surg. 2018;85(1S Suppl 2):S98-S103.

29. Sadek S, Lockey DJ, Lendrum RA, Perkins Z, Price J, Davies GE. Resuscitative endovascular balloon occlusion of the aorta (REBOA) in the pre-hospital setting: an additional resuscitation option for uncontrolled catastrophic haemorrhage. Resuscitation. 2016;107:135-138.

30. Lamhaut L, Qasim Z, Hutin A, et al. First description of successful use of zone 1 resuscitative endovascular balloon occlusion of the aorta in the prehospital setting. Resuscitation. 2018;133:e1-e2.

31. Wessels LE, Wallace JD, Bowie J, Butler WJ, Spalding C, Krzyzaniak M. Radiofrequency identification of the ER-REBOA: confirmation of placement without fluoroscopy. Mil Med. Epub 2018 Aug 6.

32. Ogura T, Lefor AK, Nakamura M, Fujizuka K, Shiroto K, Nakano M. Ultrasound-guided resuscitative endovascular balloon occlusion of the aorta in the resuscitation area. J Emerg Med. 2017;52(5):715-722.

33. Hoehn MR, Hansraj NZ, Pasley AM, et al. Resuscitative endovascular balloon occlusion of the aorta for non-traumatic intra-abdominal hemorrhage. Eur J Trauma Emerg. Surg. Epub 2018 Jun 19.

34. Shigesato S, Shimizu T, Kittaka T, Akimoto H. Intra-aortic balloon occlusion catheter for treating hemorrhagic shock after massive duodenal ulcer bleeding. Am J Emerg Med. 2015;33(3):473.e1-2.

35. Dogan EM, Beskow L, Calais F, Hörer TM, Axelsson B, Nilsson KF. Resuscitative endovascular balloon occlusion of the aorta in experimental cardiopulmonary resuscitation - aortic occlusion level matters. Shock. Epub 2018 Jul 31.

36. Daley J, Morrison JJ, Sather J, Hile L. The role of resuscitative endovascular balloon occlusion of the aorta (REBOA) as an adjunct to ACLS in non-traumatic cardiac arrest. Am J Emerg Med. 2017;35(5): 731-736.

37. Ordoñez CA, Manzano-Nunez R, Parra MW, et al. Prophylactic use of resuscitative endovascular balloon occlusion of the aorta in women with abnormal placentation: a systematic review, meta-analysis, and case series. J Trauma Acute Care Surg. 2018;84(5):809-818.

38. Sun W, Duan S, Xin G, et al. Safety and efficacy of preoperative abdominal aortic balloon occlusion in placenta increta and/or percreta. J Surg Res. 2018;222:75-84.

39. Chu Q, Shen D, He L, et al. Anesthetic management of cesarean section in cases of placenta accreta, with versus without abdominal aortic balloon occlusion: study protocol for a randomized controlled trial. Trials. 2017;18(1):240.

40. Ribeiro Junior MAF, Feng CYD, Nguyen ATM, et al. The complications associated with resuscitative endovascular balloon occlusion of the aorta (REBOA). World J Emerg Surg. 2018;13:20.

41. Ordonez CA, Nunez RM, Parra MW, et al. Common complications after the use of resuscitative endovascular balloon occlusion of the aorta (REBOA). J Am Coll Surg. 2017;225(4):S52-S53.

42. Saito N, Matsumoto H, Yagi T, et al. Evaluation of the safety and feasibility of resuscitative endovascular balloon occlusion of the aorta. $J$ Trauma Acute Care Surg. 2015;78(5):897-904.

43. Teeter WA, Matsumoto J, Idoguchi K, et al. Smaller introducer sheaths for REBOA may be associated with fewer complications. J Trauma Acute Care Surg. 2016;81(6):1039-1045.

44. Brenner M, Inaba K, Aiolfi A, et al. Resuscitative endovascular balloon occlusion of the aorta and resuscitative thoracotomy in select patients with hemorrhagic shock: early results from the American Association for the Surgery of Trauma's Aortic Occlusion in Resuscitation for Trauma and Acute Care Surgery registry. J Am Coll Surg. 2018;226(5): $730-740$.

45. Dubose JJ, Scalea TM, Brenner M, et al. The AAST prospective Aortic Occlusion for Resuscitation in Trauma and Acute Care Surgery (AORTA) registry: data on contemporary utilization and outcomes of aortic occlusion and resuscitative balloon occlusion of the aorta (REBOA). J Trauma Acute Care Surg. 2016;81(3):409-419. 
46. Brenner M, Bulger EM, Perina DG, et al. Joint statement from the American College of Surgeons Committee on Trauma (ACS COT) and the American College of Emergency Physicians (ACEP) regarding the clinical use of resuscitative endovascular balloon occlusion of the aorta (REBOA). Trauma Surg Acute Care Open. 2018;3(1):e000154.

47. Brenner M, Teeter W, Hoehn M, et al. Use of resuscitative endovascular balloon occlusion of the aorta for proximal aortic control in patients with severe hemorrhage and arrest. JAMA Surg. 2018;153(2):130-135.
48. Darrabie MD, Croft CA, Brakenridge SC, et al. Resuscitative endovascular balloon occlusion of the aorta: implementation and preliminary results at an academic level I trauma center. J Am Coll Surg. 2018;227(1): 127-133.

49. Beldowicz BC. Resuscitative endovascular balloon occlusion of the aorta in interhospital transfers: two case reports. J Endovasc Trauma Manag. 2018;2:44-48.

\section{Publish your work in this journal}

The Open Access Emergency Medicine is an international, peerreviewed, open access journal publishing original research, reports, editorials, reviews and commentaries on all aspects of emergency medicine. The manuscript management system is completely online and includes a very quick and fair peer-review system, which is all easy to use. Visit http://www.dovepress.com/testimonials.php to read real quotes from published authors. 